\title{
Philosophiques
}

\section{À propos d'une objection contre le naturalisme modéré}

\section{Martin Montminy}

Volume 30, numéro 2, automne 2003

URI : https://id.erudit.org/iderudit/008649ar

DOI : https://doi.org/10.7202/008649ar

Aller au sommaire du numéro

Éditeur(s)

Société de philosophie du Québec

ISSN

0316-2923 (imprimé)

1492-1391 (numérique)

Découvrir la revue

Citer cet article

Montminy, M. (2003). À propos d'une objection contre le naturalisme modéré. Philosophiques, 30(2), 411-415. https://doi.org/10.7202/008649ar d'utilisation que vous pouvez consulter en ligne.

https://apropos.erudit.org/fr/usagers/politique-dutilisation/ 


\title{
À propos d'une objection contre le naturalisme modéré
}

\author{
MARTIN MONTMINY \\ Université d'Ottawa \\ mmontminy@uottawa.ca
}

L'un des objectifs que se fixe Daniel Laurier dans son excellent livre L'Esprit et la nature est de montrer qu'il n'y a pas de position stable entre le naturalisme radical et le surnaturalisme, autrement dit qu'un naturalisme modéré n'est pas viable. Je vais présenter l'argument de Laurier à cet effet et soulever quelques questions à son égard.

\section{Naturalisme et normativité}

Le naturalisme affirme, en gros, que les activités humaines, y compris les activités cognitives, font partie de la nature et qu'une étude de l'être humain ne devrait pas faire appel à des entités et des principes autres que ceux qu'invoquent les sciences naturelles. L'une des principales difficultés du naturalisme est de rendre compte de la dimension normative des activités humaines. Une théorie de la connaissance, par exemple, devrait au moins pouvoir expliquer à quelles conditions nos croyances et nos raisonnements sont rationnels ou justifiés. Une théorie de la signification devrait au moins spécifier les conditions d'usage correct des énoncés de la langue. Comment réconcilier cela avec le fait que les sciences naturelles sont essentiellement descriptives? Lorsqu'une réaction chimique n'est pas conforme au modèle proposé par un chimiste, nous ne sommes pas tentés de dire que les molécules ont fait une erreur et auraient dû se comporter autrement. Mais lorsque notre objet d'étude est l'être humain, nous nous sentons autorisés (dans certains contextes) à dire que tel ou tel usage d'un mot, ou tel ou tel raisonnement, est fautif. Ainsi, une étude de l'être humain semble se démarquer d'une étude de la nature en ceci qu'elle ne se contente pas de dire comment les choses sont; elle est aussi censée dire comment elles devraient être.

Laurier distingue trois réactions possibles à ce problème. Deux de celles-ci soutiennent qu'il n'a pas de solution, autrement dit qu'il n'y a pas de normes naturelles. Le surnaturalisme renonce au naturalisme et soutient qu'une explication de la normativité ne relève pas des sciences de la nature: il y a des normes, mais celles-ci sont non naturelles. Le naturalisme radical reconnaît lui aussi qu'il n'y a pas de normes naturelles, mais plutôt que de rejeter le naturalisme, il renonce à la normativité. Le naturalisme modéré soutient qu'il y a une solution au problème que je viens de présenter, autrement dit qu'il y a des normes dans la nature.

Il est bon de distinguer deux projets naturalistes, à savoir le naturalisme épistémologique, qui concerne la justification épistémique, et le 
naturalisme intentionnel, qui porte sur le contenu intentionnel. Le reste de mon texte portera sur le second projet.

\section{L'argument de Davidson}

Le naturalisme intentionnel modéré, affirme que: (i) l'on ne peut rendre compte du contenu intentionnel sans faire appel à certaines notions normatives; (ii) les normes constitutives de l'intentionnalité sont naturelles. L'argument de Laurier contre ce point de vue tire son inspiration des écrits de Donald Davidson ${ }^{1}$. Selon Davidson, la croyance est au centre de tous les types de pensées, entendues au sens d'attitudes propositionnelles: un être ne peut avoir une attitude propositionnelle sans automatiquement avoir un certain nombre de croyances qui sont présupposées ou directement impliquées par celle-ci. Par conséquent, il est nécessaire d'avoir des croyances pour avoir des pensées, et toute condition nécessaire de la croyance est automatiquement une condition nécessaire de la pensée.

Davidson soutient par ailleurs qu'on ne peut avoir une croyance sans comprendre son contenu propositionnel. Or, comme, pour Davidson, comprendre un contenu propositionnel, c'est connaître ses conditions de vérité, un être ne peut avoir des croyances que s'il comprend que celles-ci peuvent être vraies ou fausses. Cela veut donc dire que:

(1) Pour avoir des croyances, il faut avoir les concepts de croyance et de vérité objective.

Pour Davidson, il n'y a donc pas de pensées du premier ordre sans pensées du deuxième ordre ${ }^{2}$. Davidson soutient en outre que l'interaction avec autrui est requise pour rendre possibles l'erreur et l'acquisition du concept de vérité objective. En effet, un être qui n'a pas comparé ses réponses à l'environnement avec celles d'un autre être ne peut distinguer ce qui lui semble être le cas de ce qui est le cas. Selon Davidson, seule l'interaction avec autrui permet une telle distinction. Or, cette interaction, poursuit-il, doit être linguistique, puisque le langage est la seule façon de communiquer les contenus propositionnels d'une personne à une autre. Par conséquent :

(2) La communication linguistique est requise pour l'acquisition du concept de vérité objective.

Cette thèse, combinée à la thèse (1), entraîne qu'il faut être un interprète du langage d'autrui pour avoir des croyances.

Comme le souligne Laurier, cet argument est loin d'être «contraignant ». Il semble en effet raisonnable d'attribuer à des êtres non capables

1. Voir entre autres Davidson (1984, essai 11; 1991).

2. Laurier présente un autre argument avancé par Davidson (1985) pour soutenir (1). Selon Davidson, avoir une croyance implique la possibilité de surprise. Or, pour pouvoir être surpris, il faut être capable de croire qu'une croyance antérieure était fausse. 
de pensées du deuxième ordre des représentations du monde (ou des états intentionnels) qui peuvent être vrais ou faux. Laurier propose ainsi de distinguer deux régimes d'intentionnalité: intentionnalité et méta-intentionnalité. La distinction entre les interprètes qui sont capables de pensées du deuxième ordre et les êtres qui ne peuvent avoir que des pensées du premier ordre pourrait correspondre, suggère Laurier, à la distinction entre les êtres dont les états intentionnels sont pourvus d'un contenu conceptuel et ceux dont les états intentionnels ont un contenu non conceptuel. Laurier propose donc de remplacer la thèse (1) par:

(3) Nul ne peut avoir des pensées conceptuelles sans avoir le concept de croyance correcte ou incorrecte et la capacité d'attribuer des croyances à autrui.

\section{L'argument de Laurier}

Je suis maintenant en mesure de présenter l'argument de Laurier contre le naturalisme intentionnel modéré. Laurier souligne que s'il y a des normes constitutives des contenus conceptuels, elles ne peuvent être que des normes de rationalité, ce qui entraîne que:

(4) Une explication naturaliste de la pensée conceptuelle doit comporter une explication naturaliste de la rationalité.

En outre, si les normes de rationalité sont constitutives de l'intentionnalité, un interprète doit posséder le concept de rationalité, ce qui, étant donné (3), implique que:

(5) Pour avoir des pensées conceptuelles, il faut avoir le concept de rationalité.

Mais, écrit Laurier, «s'il est vrai que nul ne peut avoir de pensée conceptuelle à moins d'avoir le concept de rationalité, alors une explication naturaliste des normes de rationalité présuppose à son tour une explication naturaliste des contenus conceptuels » (p. 144). Ainsi, selon Laurier, (5) entraîne que :

(6) Une explication naturaliste de la rationalité présuppose une explication naturaliste des contenus conceptuels.

Cette inférence me laisse perplexe. Le fait qu'on ne peut avoir des pensées conceptuelles sans avoir le concept de rationalité n'entraîne pas que l'explication de la rationalité présuppose celle de la pensée conceptuelle, mais plutôt que:

(7) Une explication naturaliste de la pensée conceptuelle présuppose une explication naturaliste des conditions de possession du concept de rationalité.

Cela dit, je vois mal comment on peut expliquer la rationalité indépendamment de la pensée conceptuelle: après tout, les normes de rationalité portent sur les relations de justification, cohérence, etc., entre pensées. 
La thèse (6) me paraît donc tout à fait plausible indépendamment de la conclusion de l'argument qu'invoque Laurier. Si cela est le cas, il devient inutile d'invoquer l'argument «non contraignant » de Davidson, ou la version de cette conclusion que propose Laurier, pour défendre (6), ce qui n'est certainement pas une mauvaise chose.

(4) et (6) entraînent que la naturalisation de l'intentionnalité et la naturalisation de la rationalité ne sont que deux facettes d'un même problème. Laurier en conclut qu' "on ne doit pas s'attendre à trouver une explication naturaliste des normes constitutives de la pensée conceptuelle, et on ne peut considérer le naturalisme intentionnel modéré $[. .$.$] qu'avec$ pessimisme »(p. 145). Si je comprends bien, l'objection de Laurier contre le naturalisme modéré est que toute tentative de naturaliser le contenu conceptuel intentionnel est vouée à tourner en rond: elle doit d'abord expliquer la rationalité pour rendre compte du contenu, et une explication de la rationalité n'est possible qu'après qu'on a fourni une explication du contenu.

\section{Quelques questions}

L'argument de Laurier soulève un certain nombre de questions. Tout d'abord, pourquoi exclut-il la possibilité d'expliquer simultanément l'intentionnalité et la rationalité en termes naturels? Une telle approche serait, me semble-t-il, compatible avec (4) et (6). Pourquoi n'est-elle pas envisageable selon Laurier?

Par ailleurs, je ne comprends pas pourquoi Laurier n'écarte pas d'emblée le naturalisme intentionnel radical. Ce point de vue extrême, je le rappelle, affirme qu'il n'y a pas de normes (naturelles ou non naturelles) relatives au contenu. Si ce point de vue est correct, je vois mal comment on peut parler de contenu mental ou linguistique. En effet, si le contenu linguistique n'est pas gouverné par des normes, on ne peut dire d'un énoncé qu'il est utilisé correctement ou non. Une notion non normative d'usage correct semble en effet incohérente. Mais si les énoncés n'ont pas de conditions d'usage correct, comment peuvent-ils être pourvus de signification? Le naturalisme radical semble donc entraîner le nibilisme intentionnel, c'est-à-dire l'idée selon laquelle il n'y a pas de contenu.

Le nihilisme intentionnel n'est pas tout à fait une thèse auto-réfutante. En effet, s'il n'y a pas de normes de vérité, les énoncés linguistiques, y compris la thèse du nihilisme intentionnel, ne sont ni vrais ni faux. La difficulté liée au nihilisme intentionnel est plutôt qu'il s'agit d'une thèse qui s'autodétruit : si cette thèse est correcte, alors son énoncé n'est qu'une suite de sons sans signification. S'il n'y a pas de signification, aussi bien cesser toute activité linguistique. La meilleure chose à faire serait peut-être d'aller méditer au Bhoutan et de se contenter de produire des sons comme "om». 


\section{Conclusion}

Reste évidemment le surnaturalisme. Malheureusement, je doute que nous disposions des ressources nécessaires pour trancher entre cette position et le naturalisme modéré. Je m'explique. Il est raisonnable de penser que les normes dépendent des phénomènes naturels (dispositions comportementales, états fonctionnels, etc.), au sens où si deux mondes sont naturellement (ou physiquement) identiques, alors ils ne diffèrent pas du point de vue des normes. Cela suggère donc qu'il existe des "principes de liaison» entre les phénomènes naturels et les normes. Sans nécessairement conduire à une réduction, de tels principes pourraient très bien permettre de dériver les normes à partir d'une description des lois, propriétés et entités naturelles d'un monde. Autrement dit, les principes de liaison spécifieraient la relation de dépendance des normes par rapport aux phénomènes naturels.

Selon ce scénario, le débat entre le naturalisme modéré et le surnaturalisme revient à la question de savoir si les principes de liaison font partie des sciences naturelles ou non. Pour répondre à cette question, il faudrait disposer de principes d'ordre supérieur qui permettraient de délimiter ce qui relève des sciences naturelles de ce qui est externe à celles-ci. Or, je doute fort qu'il existe une méthode indépendante et non arbitraire permettant d'obtenir de tels principes. Dire, par exemple, qu'un énoncé ne peut appartenir aux sciences naturelles s'il contient des termes normatifs serait une pétition de principe. Ce genre de difficultés me fait craindre que le débat entre le naturalisme modéré et le surnaturalisme ne se ramène à une querelle terminologique portant sur l'étiquette que l'on devrait assigner à certains énoncés.

\section{Bibliographie}

Davidson, Donald (1984), Inquiries into Truth and Interpretation, Oxford, Clarendon Press; trad. franç.: Enquêtes sur la vérité et l'interprétation, Nîmes, J. Chambon, 1993.

Davidson, Donald (1985), "Rational Animals», Actions and Events. Perspectives on the Philosophy of Donald Davidson (sous la dir. de E. Lepore et B. McLanghlin), Oxford, Basil Blackwell, p. 473-481.

Davidson, Donald (1991), «Epistemology Externalized», Dialectica, no 45, p. 191202.

Laurier, Daniel (2002), L'Esprit et la nature, Montréal, Presses de l'Université de Montréal. 